\title{
Helium-3 MRI diffusion coefficient: correlation to morphometry in a model of mild emphysema
}

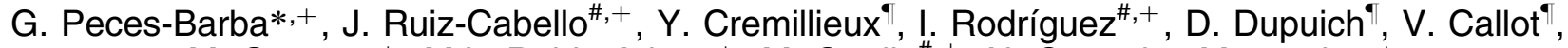 \\ M. Ortega*,+, M.L. Rubio Arbo*,+, M. Cortijo ${ }^{\#,+}$, N. Gonzalez-Mangado*,+
}

Helium-3 MRI diffusion coefficient correlation to morphometry in a model of mild emphysema. G. Peces-Barba, J. Ruiz-Cabello, Y. Cremillieux, I. Rodríguez, D. Dupuich, V. Callot, M. Ortega, M.L. Rubio Arbo, M. Cortijo, N. GonzalezMangado. (C) ERS Journals Ltd 2003.

ABSTRACT: Hyperpolarised gases have been most recently used in magnetic resonance imaging to demonstrate new image-derived pulmonary function parameters. One of these parameters is the apparent diffusion coefficient, which reflects the sizes of the structures that compartmentalise gas within the lung (i.e. alveolar space). In the present study, noninvasive parameters were compared to microscopic measurements (mean linear intercept and mean alveolar internal area).

Nonselective helium-3 gas density coronal ex vivo images and apparent diffusion maps were acquired in control and elastase-induced panacinar emphysema rats. Total lung capacity was considered the reference for both imaging experiments and lung fixation.

A mild degree of emphysema was found based on mean linear intercept $(134 \pm 25 \mu \mathrm{m})$ versus control $(85 \pm 14 \mu \mathrm{m})$. The apparent diffusion coefficients were significantly different between the two groups $\left(0.18 \pm 0.02\right.$ and $0.15 \pm 0.01 \mathrm{~cm}^{2} \cdot \mathrm{s}^{-1}$ for elastase and control, respectively).

A significant correlation between the apparent diffusion coefficient and corresponding morphometric parameters in mild emphysema was demonstrated for the first time. This study opens the possibility of estimating absolute airspace size using noninvasive techniques.

Eur Respir J 2003; 22: 14-19.
*Experimental Pulmonology Laboratory, Jiménez Díaz Foundation, Autónoma University of Madrid, and ${ }^{\#}$ Institute of Biofunctional Studies, Complutense University of Madrid, Madrid, Spain. "NMR Laboratory, National Centre for Scientific Research and Claude Bernard University, Lyon, France. ${ }^{+}$Red Respira (RTIC C03/11, FIS, ISCIII)

Correspondence: G. Peces-Barba, Departamento de Neumología Experimental, Avda Reyes Católicos 2, Fundación Jiménez Díaz, Madrid 28040, Spain

Fax: 34915504954

E-mail: gpecesba@fjd.es

Keywords: Diffusion, experimental animal model, leukocyte elastase, magnetic resonance imaging, pulmonary emphysema

Received: September 112002

Accepted after revision: February 142003

This study was supported by the European Community, Brussels, Belgium (V-framework QLG1-2000-01559) and the Ministry of Science and Technology, Madrid, Spain $(\mathrm{R}+\mathrm{D}+\mathrm{I}$ Program in Biomedicine, SAF20000115 and P4 Program TIC2000-0376).
Early imaging diagnosis of chronic obstructive pulmonary diseases (COPD) is somewhat difficult, despite modern advances in high-resolution thoracic tomography. Human panacinar emphysema is characterised by an increase in lung compliance, a decrease in expiratory flow and some alveolar destruction [1]. Elastase induces emphysema in rats with many similarities to human panacinar emphysema, and this has been widely used as a standard animal model for monitoring the initial development of the disease [2, 3]. Computed tomography (CT), and particularly thin-section CT, have both become the imaging modalities of choice for assessing the extent and pattern of alveolar destruction [4]. As an indirect measurement, CT techniques are of limited sensitivity for depicting changes associated with mild COPD and may even be inaccurate in the presence of pulmonary vascular disease [5]. Nuclear medical techniques enable rapid relatively noninvasive physiological assessment of regional ventilation, but the spatial resolution that can be achieved is limited [6]. Both $\mathrm{CT}$ and nuclear medical techniques expose the patient to ionising radiation and so their use should be limited in longitudinal studies.

ALBERT et al. [7] recently demonstrated the possibility of magnetic resonance imaging (MRI) of lung tissue filled with the hyperpolarised chemically inert noble gas xenon-129.
Since then, the technique has developed, particularly with other noble gas spin nuclei, such as hyperpolarised helium-3, and emerged as a possible alternative to established CT and nuclear medical methods for investigating physiological and physiopathological processes in pulmonary ventilation $[6,8]$. MRI-based techniques are coming into use in lung research due to their innocuous character and the optimal intrinsic spatial resolution and cross-sectional anatomical visualisation possible [9]. They are already being used to provide both static and dynamic ventilation maps of inhaled gases in humans [10, 11] and animals [12-14], and to measure gas movement within the spaces of the lung during a respiratory cycle or a breath-hold using diffusion-weighting methods [15-17].

The apparent diffusion coefficient (ADC) measured by MRI techniques quantitatively describes the relative ease or difficulty with which a molecule may diffuse within a specific medium (or mobility). This mobility is restricted when molecules are confined in a limited medium by impermeable barriers or hindered by permeable ones, such as alveolar walls. Therefore, the ADC should permit comparison of airway diameters and alveolar sizes, even if the imaging resolution is insufficient to delineate alveolar clusters directly [14].

Emphysema is characterised by significantly enlarged 
airspaces and destroyed alveolar septa [18], verified and quantified only in terms of post mortem morphometric descriptions such as mean linear intercepts $(\mathrm{Lm})$, mean alveolar internal area (AIA) or destructive index (DI) [19]. Additionally, ADC-based MRI techniques are able to detect alveolar enlargements $[14,20]$, although a direct comparison was not possible due to a difference between fixation volumes and in vivo tidal volumes [14]. Moreover, there are some unresolved issues relating to this work, such as the effect of inhaled anaesthesia, ${ }^{3} \mathrm{He} /$ oxygen interactions and the influence of gas exchange on peripheral gas mixing, variables and/or conditions, which, in an initial validation of this technique, can make interpretations difficult. In particular, the influence of ${ }^{3} \mathrm{He} / \mathrm{O}_{2}$ interactions on diffusion-weighted imaging is a very interesting subject that so far has not been sufficiently studied, and requiring additional experiments for quantification of the extent of its effect. This does not invalidate the use of this technique under live conditions, although, in the present study, it was decided to avoid gas mixing by using a simplified post mortem animal model. It might be expected in any case that the estimated ADC increases proportionally with airspace size, forming a noninvasive index of airspace enlargement and possible early emphysema diagnosis. The aim of the present study was to evaluate the potential of ADC-based MRI techniques for estimation of airspace size and diagnosis of mild panacinar emphysema in rats, including interregional correlation with microscopic morphometric results under simplified acceptable experimental conditions.

\section{Material and methods}

\section{Materials and animals}

The Animal Research Committee of each centre involved approved all animal experimentation. Male Wistar rats (180$200 \mathrm{~g}$ ) were separated into two groups: control, orotracheally instilled with saline $(n=6)$; and elastase, orotracheally instilled with 75 units $\cdot 100 \mathrm{~g} \cdot$ body weight $^{-1}$ porcine pancreatic elastase (Roche Diagnostics, Basel, Switzerland) in $0.5 \mathrm{~mL}$ saline $(\mathrm{n}=8)$. Instillation was performed as previously described [2]. After instillation and gentle animal shaking to facilitate homogenous enzyme exposure, the rats were returned to their cages, where food and water were provided ad libitum. The rats were studied 45 days after elastase or saline instillation.

\section{Magnetic resonance imaging}

At 45 days and prior to MRI, the rats were anaesthetised with sodium pentobarbital $\left(60 \mathrm{mg} \cdot \mathrm{kg} \cdot\right.$ body weight ${ }^{-1}$ i.p. $)$, tracheotomised and connected to a low-pressure transducer (Honeywell, Morristown, NJ, USA) in order to calculate inspiratory capacity (IC). The IC was obtained by very slow injection of air from the resting volume until an airway pressure of $30 \mathrm{cmH}_{2} \mathrm{O}$ was reached. The injection volume was considered to be the IC and the lungs considered to be at total lung capacity. Rats were then sacrificed by inflating the lungs to a pressure of $30 \mathrm{cmH}_{2} \mathrm{O}$ with $100 \%$ nitrogen and placed in the supine position on a dual-frequency customised radiofrequency coil inside the magnet. Before MRI experiments, rat lungs were kept inflated at IC with nitrogen $\left(\mathrm{N}_{2}\right)$ in order to avoid atelectases. Before the manoeuvres were started, animals were left for $30 \mathrm{~s}$ for lung inflation to reach functional residual capacity and then injected (an IC of $5 \mathrm{~mL}$ ${ }^{3} \mathrm{He}$ in $\mathrm{N}_{2}$ per experiment), standardised for equivalent lung capacity conditions to those in morphometric measurements (see below). All MRI experiments were performed within the first hour after sacrificing animals. Reference lung function under the same experimental conditions had already been determined in studies of ventilation distribution in small animals [21]. All MRI experiments were performed using a 17-cm diameter bore 2T Oxford magnet (Oxford Instruments, Abingdon, UK) equipped with a Surrey Medical Imaging Systems (Guildford, UK) console. High-density hyperpolarised ${ }^{3} \mathrm{He}$ was obtained by direct optical pumping of a metastable form of ${ }^{3} \mathrm{He}$, allowing generation of large quantities of polarised gas $[22,23]$. Polarised ${ }^{3} \mathrm{He}$ was produced at the Laue-Langevin Institute (Grenoble, France) and transported by car to Lyon (France) the day before MRI measurements. Polarisation ranged $10-50 \%$ (depending on the delay between optical pumping and MRI acquisition).

Ventilation (anatomical) images were obtained using coronal projections with an $8 \times 8-\mathrm{cm}$ square field of view and $256 \times 256$ reconstruction matrices acquired using a radial projection reconstruction pulse sequence. For diffusion imaging, this pulse sequence was modified with a pair of nongap diffusion sinusoidal gradients, each of $1 \mathrm{~ms}$ duration. Four coronal projections with different diffusion-weighting (the diffusion-weighting factor $(b)$ ranged $0-28,372 \mathrm{~s} \cdot \mathrm{cm}^{-2}$ ) were acquired after gas injection, during breath-hold. ADC maps were obtained from fitting the diffusion-induced signal decay on the four images on a pixel-by-pixel basis. The mean and variance of the ADCs for each animal were calculated by averaging all pixels over the entire pulmonary regions (global intrasubject mean and variance). Unavoidably, these means and variances have contributions from both large and small airways. In order to improve group interregional comparison, three regions were selected with the purpose of excluding large airways as much as possible and then the mean and variance of the ADCs for corresponding pixels in these three different pulmonary regions (mainly upper and lower right lobes and left lung) were also individually determined (regional intrasubject mean and variance) in each animal group. Finally, the mean and variance of the whole set of ADCs for control and emphysematous animals were also calculated by averaging the regional means and variances (regional intersubject means and variances) and the global means and variances (global intersubject means and variances). Additional technical details have previously been reported [24].

\section{Morphometry}

After imaging, rat chests were opened and cardiopulmonary blocks removed and fixed by filling with $10 \%$ formalin to an airway pressure of $25 \mathrm{cmH}_{2} \mathrm{O}$ for $24 \mathrm{~h}$. Sections $(5 \mu \mathrm{m})$ of each pulmonary region were stained with haematoxylin and eosin. Maps of AIAs as well as horizontal and vertical alveolar lengths, comparable to conventional $\mathrm{Lm}$, were analysed using routine Leica image software (Leica Qwin; Leica Microsystems, Cambridge, UK) on images acquired by means of a video camera (Leica DC 100; Leica Microsystems) adapted to a microscope (Olympus BX40; Olympus, Tokyo, Japan). The software automatically counts intercepts with alveolar walls following a similar scheme to the traditional manual system [25], although it increases the accuracy of the method by computing traces from many more overlaid vertical and horizontal lines.

\section{Statistical analysis}

Nonparametric Mann-Whitney U-tests and Wilcoxon signed-rank tests were employed to compare animal groups 

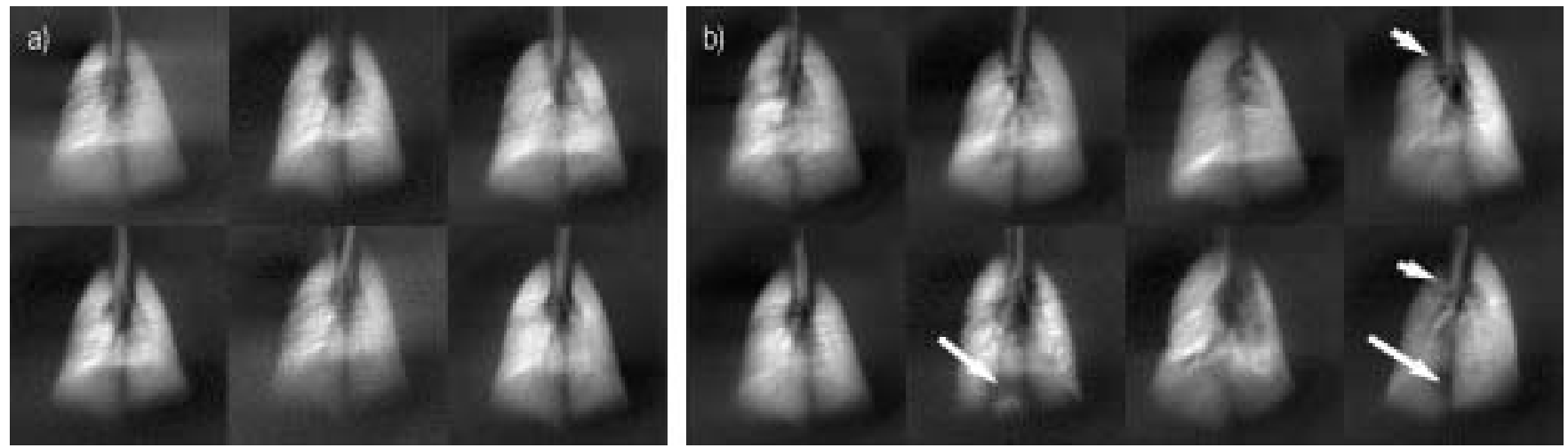

Fig. 1.-Ventilation images of all: a) control; and b) elastase-induced panacinar emphysema rats studied (the regional classification data are presented in table 1). Arrows indicate minor or subtle ventilation inhomogeneities in the elastase-treated animals.

and pulmonary regions, respectively. Analysis of correlation between MRI and morphometric measurements was performed using the nonparametric Spearman's test. BlandAltman analysis was finally applied to compare the methods [26]. Data are presented as mean \pm SD.

\section{Results}

Figure 1 shows ${ }^{3} \mathrm{He}$ gas spin density (ventilation) images from both control $(n=6)$ and emphysematous $(n=8)$ rats. Generally speaking, despite substantial and well-known differences in lung size and morphology, a very homogenous distribution pattern was observed in both groups, i.e. no substantial differences in terms of gas distribution were demonstrated using this technique. This is perhaps a predictable result, at least with mild elastase-induced emphysema, which commonly exhibits homogeneously distributed airspace enlargement, as occurred in the present case (fig. 2).

After acquisition of these ventilation images, four new fast images were obtained from the same animals with different diffusion-weightings, which allowed calculation of ADC maps by linear data fitting on a pixel-by-pixel basis (see Magnetic resonance imaging section). The ADCs in these maps were always homogenously distributed, with values that typically ranged $0.14-0.30 \mathrm{~cm}^{2} \cdot \mathrm{s}^{-1}$, depending on the animal and/or group. No visible differences in signal intensity were observed between the ADC maps of both groups of animals. The global intrasubject means and variances for control and emphysematous animals were compared using the Mann-Whitney U-test, and showed no significant differences ( $p>0.05)$. This result could be due to the large-airway contribution to the ADC means. The calculations were then repeated using manually delimited regional intrasubject data that were mainly introduced to exclude these large-airway contributions. Moreover, they permit better correlation with regional morphometric data. With this aim, after finishing MRI experiments, regional histological data (in terms of $\mathrm{Lm}$ and AIA) were obtained for both groups of animals and these data were also used for comparison to ADC results.

The IC was measured and shown apparently to have increased (although not significantly) in panacinar (14.7士 $1.2 \mathrm{~mL})$ versus control $(13.3 \pm 1.9 \mathrm{~mL})$ animals. Table 1 summarises the data for the three spatially considered ranges (spatial selection based on rat lung structure) and classification of these animals within the experimental intervals of regional intersubject $\mathrm{ADC}$ and morphometric results. Owing to the possible influence of the instillation process itself, all of these measurements (MRI-derived and histological) were evaluated for the possible influence of regional distribution, despite the unsatisfactory (although almost inevitable in a reasonable experimental time) dorsolateral projections used, which surely incorporate some data from conducting airways. A first area was selected in the upper right lobe, a second from the distal area of the lower right lobe, and, finally, a third in the medial/distal part of the left lung (fig. 3). These regions of interest of similar volume were manually outlined with the aid of a computer in the reconstructed ADC maps of both animal groups, and the regional intrasubject ADC data, calculated for each area and animal, were compared to corresponding morphological data obtained in selected fields in the same areas. For morphometry, data (both mean AIA and $\mathrm{Lm}$ ) were averaged from a minimum of $27 \pm 2$ microscopic fields located in identical areas to the MRI selected regions. In table 1 , the first range of values was chosen considering only results (ADC and morphometric) from control animals, and then two additional classes were arbitrarily added to categorise animal behaviour versus elastase treatment. The regional intersubject mean ADC (MRI) and alveolar parameters (morphometry) are summarised in table 2, and reveal a significant difference between the two animal groups (control and elastase rats).
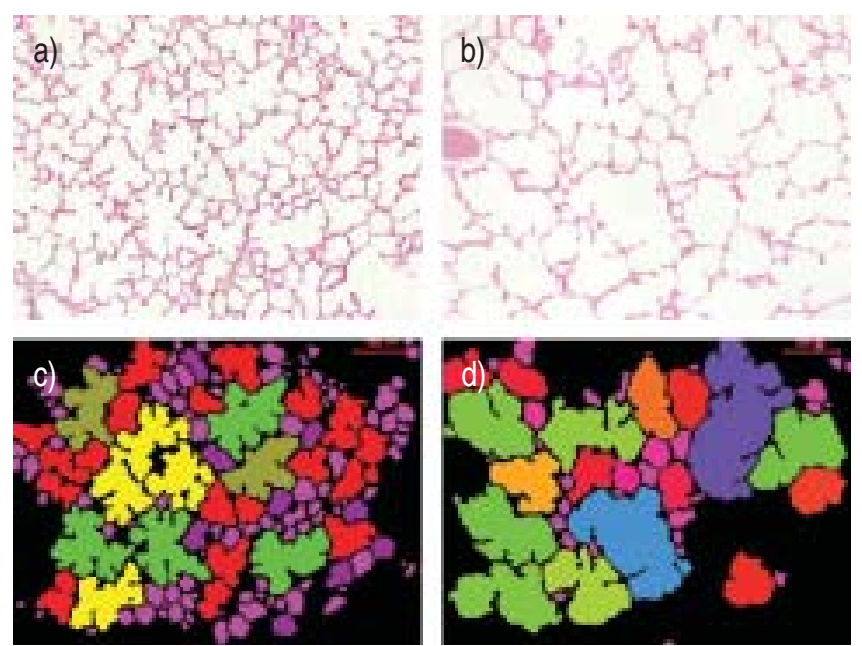

Fig. 2.- Representative photomicrographs of lung sections from: a) control; and b) elastase-induced panacinar emphysema rats (Haematoxylin and eosin stain). c, d) Automated computer procedure for quantifying the corresponding airspaces and mean linear intercepts. This method classifies units within an optional range of mean alveolar area, and shows each pattern identified as a different colour. Note the airspace enlargement shown in lung sections from the elastase-treated rats. Internal scale bars $=200 \mu \mathrm{m}$. 
Table 1.- Regional classification of panacinar" rat lungs in terms of morphometric and magnetic resonance imaging parameters

\begin{tabular}{|c|c|c|c|c|c|c|c|c|c|c|c|c|}
\hline \multirow[t]{3}{*}{ Rat } & \multicolumn{4}{|c|}{ Lower right lobe } & \multicolumn{4}{|c|}{ Upper right lobe } & \multicolumn{4}{|c|}{ Left lung } \\
\hline & \multicolumn{2}{|c|}{$10^{-2} \mathrm{AIA}$} & \multicolumn{2}{|c|}{$\mathrm{ADC}$} & \multicolumn{2}{|c|}{$10^{-2} \mathrm{AIA}$} & \multicolumn{2}{|c|}{$\mathrm{ADC}$} & \multicolumn{2}{|c|}{$10^{-2} \mathrm{AIA}$} & \multicolumn{2}{|c|}{$\mathrm{ADC}$} \\
\hline & $\mu \mathrm{m}^{2}$ & Class & $\mathrm{cm}^{2} \cdot \mathrm{s}^{-1}$ & Class & $\mu \mathrm{m}^{2}$ & Class & $\mathrm{cm}^{2} \cdot \mathrm{s}^{-1}$ & Class & $\mu \mathrm{m}^{2}$ & Class & $\mathrm{cm}^{2} \cdot \mathrm{s}^{-1}$ & Class \\
\hline Control & $87 \pm 38$ & 1 & $0.15 \pm 0.01$ & 1 & $77 \pm 28$ & 1 & $0.15 \pm 0.01$ & 1 & $84 \pm 39$ & 1 & $0.16 \pm 0.01$ & 1 \\
\hline e. 1 & $314 \pm 50$ & 3 & $0.17 \pm 0.04$ & 2 & $302 \pm 56$ & 3 & $0.18 \pm 0.05$ & 3 & $300 \pm 29$ & 3 & $0.19 \pm 0.04$ & 3 \\
\hline e. 2 & $271 \pm 67$ & 3 & $0.16 \pm 0.02$ & 2 & $233 \pm 58$ & 3 & $0.17 \pm 0.02$ & 2 & $312 \pm 87$ & 3 & $0.18 \pm 0.03$ & 2 \\
\hline e. 3 & $244 \pm 27$ & 3 & $0.21 \pm 0.05$ & 3 & $255 \pm 30$ & 3 & $0.19 \pm 0.06$ & 3 & $234 \pm 56$ & 3 & $0.19 \pm 0.05$ & 3 \\
\hline e. 4 & $160 \pm 45$ & 2 & $0.17 \pm 0.06$ & 2 & $110 \pm 24$ & 1 & $0.15 \pm 0.05$ & 1 & $74 \pm 18$ & 1 & $0.15 \pm 0.03$ & 1 \\
\hline e.5 & $105 \pm 29$ & 1 & $0.16 \pm 0.03$ & 2 & $95 \pm 20$ & 1 & $0.16 \pm 0.02$ & 2 & $82 \pm 22$ & 1 & $0.17 \pm 0.02$ & 2 \\
\hline e. 6 & $188 \pm 61$ & 2 & $0.18 \pm 0.06$ & 2 & $171 \pm 51$ & 2 & $0.18 \pm 0.06$ & 2 & $164 \pm 20$ & 2 & $0.16 \pm 0.04$ & 1 \\
\hline e.7 & $239 \pm 103$ & 3 & $0.19 \pm 0.04$ & 3 & $343 \pm 92$ & 3 & $0.17 \pm 0.04$ & 2 & $124 \pm 16$ & 1 & $0.20 \pm 0.04$ & 3 \\
\hline e. 8 & $325 \pm 67$ & 3 & $0.17 \pm 0.05$ & 2 & $322 \pm 54$ & 3 & $0.21 \pm 0.09$ & 3 & $207 \pm 81$ & 3 & $0.15 \pm 0.04$ & 1 \\
\hline
\end{tabular}

Data are presented as mean \pm SD. Spatial selection was based on rat lung structure. For the control, intersubject data are given since there is very little difference among these data. AIA: alveolar internal area; ADC: apparent diffusion coefficient. \#: codes (e.1, e.2, etc.) are as in figure 1. ": intrasubject mean AIAs are divided into three classes as suggested by the values observed: $1: 7,000 \leqslant$ AIA $<15,000 ; 2: 15,000 \leqslant$ AIA $<20,000 ;$ and $3:>20,000 \mu \mathrm{m}^{2}$. Similarly intrasubject ADCs are divided into: $1: 0.14 \leqslant \mathrm{ADC}<0.16 ; 2: 0.16 \leqslant \mathrm{ADC}<0.18 ;$ and $3:>0.18 \mathrm{~cm}^{2} \cdot \mathrm{s}^{-1}$.

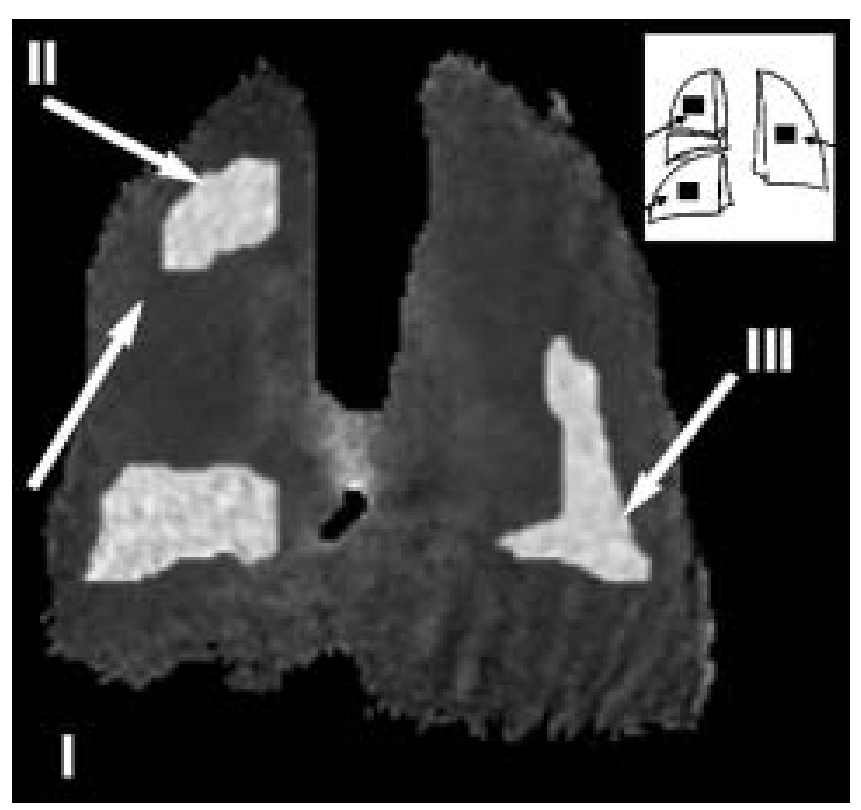

Fig. 3. - Apparent diffusion coefficient (ADC) map showing the three computer-selected areas (I-III) for comparison to morphometric data. ADCs were extracted as the mean \pm SD of all pixels included in each region of interest. Areas were selected considering the typical multilobular structure of rat lung (inset).

A regional comparison should permit demonstration of possible significant effects and interactions among animal groups (control and emphysematous) with localisation (due to a possible effect of elastase treatment) and/or parameter (ADC, mean AIA and Lm). The regional intersubject ADC

Table 2. - Regional intersubject apparent diffusion coefficient $(A D C)$, alveolar internal area (AIA) and mean linear intercept (Lm) for control and elastase-treated rats

\begin{tabular}{lcclc}
\hline & $\begin{array}{c}\text { Subjects } \\
\mathrm{n}\end{array}$ & $\begin{array}{c}\mathrm{ADC} \\
\mathrm{cm}^{2} \cdot \mathrm{s}^{-1}\end{array}$ & $\begin{array}{c}10^{-2} \mathrm{AIA} \\
\mu \mathrm{m}^{2}\end{array}$ & $\begin{array}{c}\mathrm{Lm} \\
\mu \mathrm{m}\end{array}$ \\
\hline Control & 6 & $0.15 \pm 0.01$ & $101 \pm 26$ & $85 \pm 14$ \\
Elastase & 8 & $0.18 \pm 0.02 * * *$ & $216 \pm 87^{* * *}$ & $134 \pm 25^{* * *}$ \\
\hline
\end{tabular}

Data are presented as mean \pm SD. $* * *: p<0.001$ versus control (MannWhitney U-test). and morphometric values obtained in control and emphysematous rats were significantly increased in diseased animals (Mann-Whitney test, $\mathrm{p}<0.001$ for both comparisons), confirming both the enlargement of distal airspaces with elastase treatment and the presence of less diffusion restriction, respectively. No significant differences were observed among localisation data in either animal group (Wilcoxon test, $\mathrm{p}>0.05$ ).

Direct comparison of regional ADC and morphological data (fig. 4a) revealed significant linear correlation (Spearman's two-sided correlation coefficient 0.71 for AIA, $\mathrm{p}<0.001$; including data from all animals and from the three selected areas). Similar results were obtained when comparing ADC to $\mathrm{Lm}(0.70, \mathrm{p}<0.001)$. Additionally, as expected, high correlation between mean AIA and $\mathrm{Lm}^{2}$ (and $\mathrm{Lm}$ ) was observed $(\mathrm{p}<0.001$, Spearman's two-sided correlation coefficient 0.99$)$. Finally, Bland-Altman concordance analysis was carried out in order to support the previously shown statistical results. Figure $4 \mathrm{~b}$ provides information on the absence of evident bias between MRI-derived (ADC) and morphometric measurements (mean alveolar area).

\section{Discussion}

The present study demonstrates that ADC assessment by ${ }^{3} \mathrm{He}$ MRI enables detection of mild emphysema and satisfactory correlation with microscopic morphometric measurements in a model of elastase-induced emphysema in rats.

An increase in ADC in emphysematous rats is to be expected because the alveolar dimensions increase with the partial destruction of lung architecture, and, therefore, the gas is subject to less restriction to diffusion. ADC maps in control and emphysematous rats show uniform ADC distribution, probably related to a uniform airspace (enlarged in treated animals). Only evaluation of regional ADCs between the upper and lower portions of the right lobe, and the left lung, revealed significantly higher ADCs in panacinar-like animals with respect to healthy rats. The homogeneous ADC was not surprising, and was consistent with the uniformly altered morphology evident upon morphometric measurements, despite the surely complex regional distribution of elastase solution in the pulmonary airways during the instillation procedure [27].

Taking into account the elastase concentration administered and the calculated $\mathrm{Lm}(134 \mu \mathrm{m})$, the emphysema can be classified as of mild intensity [2]. Forty-five days after 

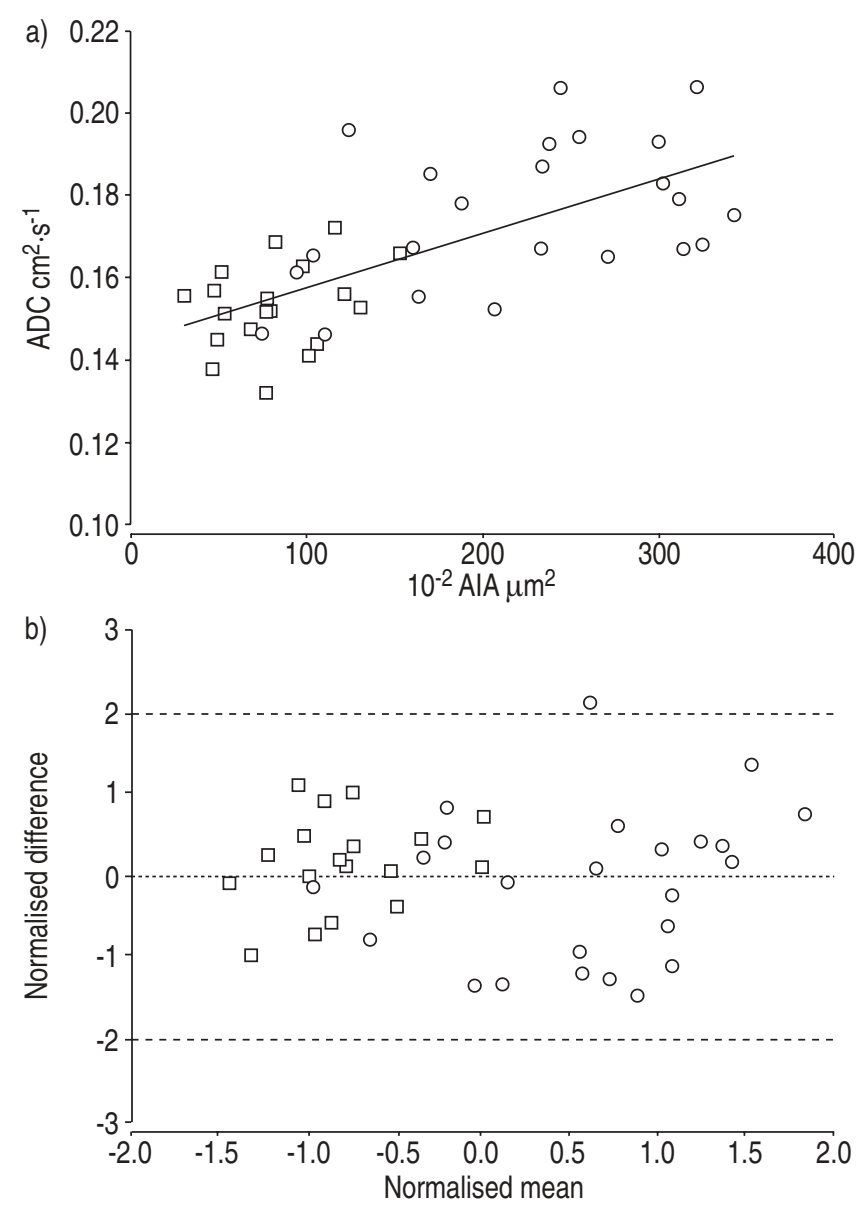

Fig. 4.-a) Correlation between mean alveolar internal area (AIA) and apparent diffusion coefficient (ADC) including mean data from all animals and the three selected areas (regional intrasubject means). b) Bland-Altman plot of the normalised regional intrasubject data collected by magnetic resonance imaging (ADC) and morphometry (mean AIA). $\square$ : control; $\bigcirc$ : elastase-induced panacinar emphysema; ......... zero mean difference; ----: 2SD of difference between corresponding normalised paired measurements.

elastase instillation, lung function and ventilation distribution tests demonstrated significant changes with respect to control animals [3], with minimum inflammatory haemorrhagic component typically present in the first days after instillation. The mean ADC data obtained in the present experiments were very similar to those published previously $\left(0.14 \mathrm{~cm}^{2} \cdot \mathrm{s}^{-1}\right.$ for control animals and $0.17 \mathrm{~cm}^{2} \cdot \mathrm{s}^{-1}$ for elastasetreated animals at end-expiratory volume) [14]. However, direct comparison with these values is difficult due to significant differences to the present experimental conditions. Thus $\mathrm{Lm}$ were higher than those published by the same authors [14], who demonstrated means of $76.5 \mu \mathrm{m}$ (control) and $97.3 \mu \mathrm{m}$ (elastase). Among other reasons, these differences could be attributed to the longer study period after elastase instillation in the present case or simply to the different experimental conditions used. CHEN et al. [14] could not make a direct comparison between $\mathrm{Lm}$ and diffusion coefficient due to a difference between the fixation volumes and the tidal volumes at which MRI experiments were carried out. In the present case, MRI experiments and lung fixation were performed at total lung capacity, which made such comparison possible.

It was hypothesised that this initial stage of the disease is where MRI-based techniques might be very valuable, since
CT-based techniques have been shown to be poorly sensitive. Moreover, comparisons [28] or correlations [29, 30] between CT and microscopic morphometry usually fail in mild emphysema. Several morphological criteria have been suggested for the microscopic definition of emphysema, including an increase in AIA, Lm or DI. All of these definitions show poor correlation with macroscopic emphysema evaluation in humans [19], making firm diagnosis of this disease very subjective [31]. In addition, correlations between macroscopic emphysema and CT scores also failed in mild emphysema [32]. High-resolution CT studies have shown significant correlations with microscopic emphysema parameters [33], but not if only data from subjects with mild emphysema are considered. This technique has not yet been standardised and the imaging quantification of pulmonary emphysema is still not resolved [34].

Experimental settings (for determining morphometric and functional data) in panacinar emphysema have been welldocumented and classified [2,3]. The present model facilitates direct comparison between morphometry and airspace MRI with well-preserved ventilation distribution homogeneity in the lung periphery [2,3]. Moreover, the present authors' studies have been performed on dead animals in order to minimise the effect of ${ }^{3} \mathrm{He} / \mathrm{O}_{2}$ interactions and the influence of gas exchange on peripheral gas mixing [21, 35]. These two conditions avoid possible artefacts and misinterpretation of MRI diffusion-weighting methods. In addition to this, all manoeuvres started from the same pre-inspiratory lung volume, identical inspiratory volume and equal volume history in order to maintain the same ventilation distribution conditions.

Anatomical (ventilation or gas distribution images) coronal projections were, in general, not very demonstrative of emphysematous disease in the present study. It is well known that the panacinar elastase rat model induces regular distribution of airspace enlargement. Generally speaking, the present images were very similar and fairly uniform, as reported previously by other authors [13], although some minor or subtle ventilation inhomogeneities can be appreciated in some cases (fig. 1). This result strengthens the conclusion of these same authors, who pointed out that ${ }^{3} \mathrm{He}$ gas density or helium anatomical imaging, at its present level of technical development, is only sensitive to the density of the inhaled gas within the lung and may not be an optimal method for the detection of early-stage emphysema. Nevertheless, much more investigation and further results, particularly those derived from less homogeneous emphysema ventilation patterns that are closer to human characteristics than the elastase model, should provide additional data on lung structure. Additionally, signal attenuation due to diffusion is prohibitively high in this type of imaging (diffusion attenuation factor 0.98 for a 2,000- $\mu$ s selective pulse and 5-mm slice, reduced to 0.67 for a $1-\mathrm{mm}$ slice and identical parameters; additional signal attenuation is derived from losses in a time constant describing loss in the net magnetisation vector in the transverse plane due to dephasing from spin/spin interactions, diffusion, magnetic field inhomogeneities and other elements $\left(T_{2}{ }^{*}\right)$ due to the consequent longer echo times) with small animals, when the strong gradients needed for slice selection and small fields of view are used. For this reason, in micro-imaging applications, only projections (no slice-selection gradient) or thicker slices are currently acquired (e.g. 5-mm slice thickness in [13]), which leads to superposition of parenchyma, reductions in the final resolution and complex data interpretation. Future extensions of these studies include time courses following the progression of $\mathrm{ADC}$ in this and other animal models. It will equally be very interesting to study other animals with different panacinar and centriacinar emphysema patterns, which may be the basis for future clinical studies 
with smoking populations, perhaps with incipient emphysema, although with normal lung function and imaging results.

In summary, for the first time, satisfactory correlation between apparent diffusion coefficients obtained by helium-3 diffusion magnetic resonance imaging and the corresponding morphometric data have been demonstrated in three regions of rat lung with mild emphysema. To the best of the present authors' knowledge, this is one of the most favourable morphometry/imaging correlations in mild emphysema. These measurements may be important as a calibration standard for the absolute determination of airspace size via apparent diffusion coefficient measurement.

Acknowledgements. The authors thank C. Santisteban for statistical advice and corrections.

\section{References}

1. Kim WD, Eidelman DDH, Izquierdo JL, Ghezzo H, Saetta MP, Cosio MG. Centrilobular and panlobular emphysema in smokers. Distinct morphologic and functional entities. Am Rev Respir Dis 1991; 144: 1385-1390.

2. González-Mangado N, Peces-Barba G, Cabanillas JJ, Renedo G, Verbänck S, Paiva M. Effect of single breath washout and lung function of elastase induced emphysema in rats. Am J Respir Crit Care Med 1993; 148: 975-981.

3. Rubio ML, Sánchez-Cifuentes MV, Peces-Barba G, Verbanck S, Paiva M, González Mangado N. Intrapulmonary gas mixing in panacinar- and centriacinar-induced emphysema in rats. Am J Respir Crit Care Med 1998; 157: 237-245.

4. Cleverley JR, Müller NL. Advances in radiologic assessment of chronic obstructive pulmonary disease. Clin Chest Med 2000; 21: 653-663.

5. Worthy SA, Müller NL, Hartman TE, Swensen SJ, Padley SP, Hansell DM. Mosaic attenuation pattern on thin-section CT scans of the lung: differentiation among infiltrative lung, airway, and vascular diseases as a cause. Radiology 1997; 205: 465-470.

6. Mayo JR, Hayden ME. Hyperpolarized helium-3 diffusion imaging of the lung. Radiology 2002; 222: 8-11.

7. Albert M, Cates GD, Driehuys B, et al. Biological magnetic resonance imaging using laser polarized ${ }^{129} \mathrm{Xe}$. Nature 1994; 370: 199-201.

8. Beardsley T. Seeing the breath of life. Sci Am 1999; 280: 24-26.

9. Kauczor HU, Chen XJ, van Beek EJR, Schreiber WG. Pulmonary ventilation imaged by magnetic resonance: at the doorstep of clinical application. Eur Respir J 2001; 17: $1008-1023$.

10. Kauczor HU, Hofmann D, Kreitner KF, et al. Normal and abnormal pulmonary ventilation: visualization at hyperpolarized He-3 MR imaging. Radiology 1996; 201: 564-568.

11. MacFall JR, Charles HC, Black RD, et al. Human lung air spaces: potential for MR imaging with hyperpolarized He-3. Radiology 1996; 200: 553-558.

12. Black RD, Middleton HL, Gates GD, et al. In vivo He-3 MR images of guinea pig lungs. Radiology 1996; 199: 867-870.

13. Eberle B, Weiler N, Markstaller K, et al. Analysis of intrapulmonary $\mathrm{O}_{2}$ concentration by MR imaging of inhaled hyperpolarized helium-3. J Appl Physiol 1999; 87: 2043-2052.

14. Chen XJ, Hedlund LW, Möller HE, Chawla MS, Maronpot RR, Johnson GA. Detection of emphysema in rat lungs by using magnetic resonance measurements of ${ }^{3} \mathrm{He}$ diffusion. Proc Natl Acad Sci USA 2000; 97: 11478-11481.
15. Saam BT, Yablonskiy DA, Kodibagkar VD, et al. MR imaging of diffusion of 3-He gas in healthy and diseased lungs. Magn Reson Med 2000; 44: 174-179.

16. Salerno M, de Lange EE, Altes TA, Truwit JD, Brookeman JR, Mugler JP III. Emphysema: hyperpolarized helium-3 diffusion MR imaging of the lungs compared with spirometric indexes - initial experience. Radiology 2002; 222: 252-260.

17. Yablonskiy DA, Sukstanskii AL, Leawoods JC, et al. Quantitative in vivo assessment of lung microstructure at the alveolar level with hyperpolarized ${ }^{3} \mathrm{He}$ diffusion MRI. Proc Natl Acad Sci USA 2002; 99: 3111-3116.

18. National Institutes of Health. The definition of emphysema: report of a National Heart, Lung and Blood Institute, Division of Lung Diseases, workshop. Am Rev Respir Dis 1985; 132: 182-185.

19. Thurlbeck WM. The internal surface area of nonemphysematous lungs. Am Rev Respir Dis 1967; 95: 765-773.

20. Chen XJ, Möller HE, Chawla MS, et al. Spatially resolved measurements of hyperpolarized gas properties in the lung in vivo. Part I: diffusion coefficient. Magn Reson Med 1999; 42: 721-728.

21. Gonzalez-Mangado N, Peces-Barba G, Verbanck S, Paiva M. Single breath washout experiments in rat lungs: effects of diffusion-convection interaction on the slope of the alveolar plateau. J Appl Physiol 1991; 71: 847-854.

22. Colegrove FD, Schearer LD, Walters GK. Polarization of ${ }^{3}$ He gas by optical pumping. Phys Rev 1963; 132: 2561-2572.

23. Eckert G, Heil W, Meyerhoff M, et al. A dense polarized ${ }^{3} \mathrm{He}$ target based on compression of optically pumped gas. Nucl Instrum Methods Phys Res A 1992; 320: 53-65.

24. Cremillieux Y, Berthezene $\mathrm{Y}$, Humblot $\mathrm{H}$, et al. A combined ${ }^{1} \mathrm{H}$ perfusion $/{ }^{3} \mathrm{He}$ ventilation NMR study in rat lungs. Magn Reson Med 1999; 41: 645-648.

25. Dunnill MS. Quantitative methods in the study of pulmonary pathology. Thorax 1962; 17: 320-328.

26. Bland JM, Altman DG. Statistical methods for assessing agreement between two methods of clinical measurement. Lancet 1986; 1: 307-310.

27. Cassidy KJ, Bull JL, Glucksberg MR, et al. A rat lung model of instilled liquid transport in the pulmonary airways. J Appl Physiol 2001; 90: 1955-1967.

28. Remy-Jardin M, Remy J, Gosselin B, Copin MC, Wurtz A, Duhamel A. Sliding thin slab, minimum intensity projection technique in the diagnosis of emphysema: histopathologicCT correlation. Radiology 1996; 200: 665-671.

29. Spouge D, Mayo JR, Cardoso W, Muller NL. Panacinar emphysema: CT and pathologic findings. J Comput Assist Tomogr 1993; 17: 710-713.

30. Kuwano K, Matsuba K, Ikeda T, et al. The diagnosis of mild emphysema. Correlation of computed tomography and pathology scores. Am Rev Respir Dis 1990; 141: 169-178.

31. Thurlbeck WM, Muller NL. Emphysema: definition, imaging and quantification. Am J Roentgenol 1994; 163: 10171025 .

32. Miller RR, Muller NL, Vedal S, Morrison NJ, Staples CA. Limitations of computed tomography in the assessment of emphysema. Am Rev Respir Dis 1989; 139: 980-983.

33. Gevenois PA, de Vuyst P, de Maertelaer V, et al. Comparison of computed density and microscopic morphometry in pulmonary emphysema. Am J Respir Crit Care Med 1996; 154: 187-192.

34. Madani A, Keyzer C, Gevenois PA. Quantitative computed tomography assessment of lung structure and function in pulmonary emphysema. Eur Respir J 2001; 18: 720-730.

35. Verbanck S, Gonzalez-Mangado N, Peces-Barba G, Paiva M. Multiple breath washout experiments in rat lungs: an evaluation of intraregional ventilation distribution. $J \mathrm{Appl}$ Physiol 1991; 71: 847-854. 